\title{
An Efficient Object Tracking Algorithm of Sports Video
}

\author{
Hongyuan Guo ${ }^{1, a}$ \\ ${ }^{1}$ Gansu Normal University for Nationalities, Hezuo, gansu, 747000, China \\ aHongyuanGuo@163.com
}

Keywords: sports video; particle filter; mean- shift; hybrid tracking; algorithm

\begin{abstract}
This paper studies the moving object tracking technology for sports video and puts forward an optimized hybrid tracking method. First, particle filter algorithm is adopted to predict the position of moving target, and then compute comparability between object model and object on the estimated position, if the comparability is less than a certain threshold value, we consider the object's dynamic model has changed and need to modify the dynamic model; if the comparability is more than a certain threshold value, we consider the object's dynamic model has not changed and don't need to modify the dynamic model, in this way we can find the dynamic model of optimization. Finally we use this dynamic model of optimization in mean- shift algorithm, and compute object's true position.
\end{abstract}

\section{Introduction}

Moving object detection and tracking is an important subject in the field of vision. Its core idea is to use the technology of image processing and video analysis to capture the moving objects quickly and accurately. At present, the most of the target tracking algorithms are based on the template matching method of motion estimation. So the motion estimation has a direct impact on the accuracy of the tracking results.

Literature [1] proposes a color based particle filter tracking algorithm, which uses color histogram as the feature to track the moving object. Literature [2] proposes a novel tracking algorithm based on the contour of the target, the use of optical flow method to track the target contour, but the optical flow method is computationally complex, low accuracy, and the interference is poor, vulnerable to the impact of noise. Literature [3] proposes a kernel mean shift algorithm. The algorithm has high computational complexity and low precision. However, due to the limitations of convergence, the algorithm can only predict the position of the target with the real position of the target $^{[4]}$.

In sports video, the moving speed of the moving object is fast, and the movement speed often has the huge change, the general movement model is difficult to accurately forecast the movement goal the approximate position ${ }^{[5]}$. Therefore, many of the above algorithms are difficult to achieve good results in the sports video, the algorithm is given in this paper is a good solution to the above problems $^{[6]}$. First, particle filter algorithm is adopted to predict the position of moving target, through the comparison of the similarity of the predicted target position between the target model, when the similarity is less than a certain threshold value that target motion model occurred fundamental changes, need to enable the new motion model; when the similarity is greater than a certain threshold value that the motion model of the target did not happen big change, do not need to enable the new motion model. Then the kernel based mean shift algorithm is used to get the accurate position of the target $^{[7]}$.

\section{Target model}

Target tracking algorithm relies heavily on the choice of the target model. At present, there are two kinds of commonly used target models: One is based on the outline of the target, the other is based on the color characteristics of the target. The advantage of contour feature is high precision, 
the disadvantage is that the computation is complex, the disturbance is poor, and the advantage of the color feature has strong robustness, strong noise and good anti blocking property. Therefore, this paper uses color histogram as the object model. The kernel function is used to deal with the color histogram, and the color distribution of the target is extracted.

The assumption is that the target is a rectangular window of center for $y$, the window width is $h$, the pixel position of the target in the image is $\left(x_{i}\right) i=1, \ldots, n$. If the target model is $m$ histogram, the normalized post target color distribution can be expressed as:

$$
\begin{aligned}
& q(y)=\left\{q_{u}(y) \quad u=1, \ldots, m\right\} \\
& \sum_{u=1}^{m} q_{u}(y)=1 \\
& \mathrm{q}_{u}(y)=c_{h} \sum_{i=1}^{n} g\left(x_{i}\right) \sigma\left[b\left(x_{i}\right)-u\right]
\end{aligned}
$$

Among them, $b\left(x_{\mathrm{i}}\right)$ is the index value of the pixel point $x_{\mathrm{i}}$ in the M histogram. Function $g\left(x_{i}\right)$ is used to represent the weight of the pixel point $x_{\mathrm{i}}$ in the color distribution of the object.

$$
\begin{aligned}
& \mathrm{g}\left(x_{i}\right)=k\left(\left\|\frac{x_{i}-x_{0}}{h}\right\|^{2}\right) \\
& c_{h}=\frac{1}{\sum_{i=1}^{n} k\left(\left\|\frac{x_{i}-x_{0}}{h}\right\|^{2}\right)}
\end{aligned}
$$

The target model for the $m$ value of normalized histogram, color histogram target model is $p=$ $\left\{p_{\mathrm{u}}\right\}, u=1, \ldots, m$, the normalized color histograms of candidate target in the location $y$ is $q(y)=\left\{q_{u}(y) \quad u=1, \ldots, m\right\}$. The similarity of the target model and the candidate target is:

$$
\rho[\mathrm{p}, q(y)]=\sum_{u=1}^{m} \sqrt{p_{u}} q_{u}(y)
$$

\section{Particle filter algorithm}

In this paper, the particle filter algorithm is as follows:

(1) Select $n$ sample from the sample set $\mathrm{s}_{\mathrm{t}-1}=\left\{S_{t-i}^{n}\right\} \quad n=1, \ldots, N$ to obey the probability distribution $\pi_{t-1}^{n}$.

(1) Calculation the cumulative probability distribution $c_{y-1}$.

(2) Generating $\mathrm{N}$ random numbers obeying the uniform distribution $r_{n} \in[0,1]$.

(3) In the set $\left\{\mathrm{c}_{\mathrm{t}-1}^{(\mathrm{j})}\right\}$ selected to the minimum $j$ meet conditions $\mathrm{c}_{\mathrm{t}-1}^{(\mathrm{j})} \geq \mathrm{r}_{n}$.

(2) By using the motion equations $E\left[S \sum_{n=1}^{N} S^{n} \pi^{n}\right]$ from the collection $\mathrm{s}_{t-1}=\left\{S_{t-1}^{(n)}\right\}$ to produce an image of the sample set $\mathrm{S}_{t}=\left\{S_{t}^{(n)}\right\}$. 
(3) The location of the target in the next frame image:

(1) Calculate the similarity of each sample point and the target model $\rho\left[\mathrm{p}, q\left(\mathrm{~s}_{t}^{(n)}\right)\right]$

(2) Calculate the probability weight of each sample point $\pi_{t}^{n}$.

$\pi_{t}^{n}=\frac{1}{\sqrt{2 \pi \sigma}} e^{-\frac{\left(1-\rho\left[p, q\left(S_{t}^{(n)}\right)\right]\right)}{2 \sigma^{2}}}$

(3) Expected the estimate value of target position:

$$
E\left[S_{t}\right]=\sum_{n=1}^{N} \pi_{t}^{n} S_{t}^{(n)}
$$

\section{Mean shift algorithm}

Mean shift algorithm is an iterative algorithm for solving optimization problems. In this paper, we assume that the initial position of the moving object is $y_{0}$ by particle filter. The similarity of the candidate target $\mathrm{y}_{0}$ and the target model is:

$$
\rho[\mathrm{p}, q(y)]=\sum_{u=1}^{m} p_{u} q_{u}(y)
$$

We might as well assume that $\left\{x_{i}\right\} i=1, \ldots, n$ for a set of sample collection, $y_{0}$ is the initial cluster center of the set, $w_{i}$ is fuzzy membership degree function of each data point $x_{i}$, we use fuzzy clustering method to solve the clustering center point $y^{[8]}$ :

$$
\mathrm{y}=\frac{\sum_{i=1}^{n} x_{i} W_{i} g\left(\left\|y_{0}-x_{i}\right\|^{2}\right)}{\sum_{i=1}^{n} W_{i} g\left(\left\|y_{0}-x_{i}\right\|^{2}\right)}
$$

The following is the algorithm steps of mean shift algorithm:

(1) Calculate the similarity between the initial target and the target model: $\rho\left[\mathrm{p}, q\left(y_{0}\right)\right]=\sum_{u=1}^{m} p_{u} q_{u}\left(y_{0}\right)$

(2) According to $\mathrm{y}=\frac{\sum_{i=1}^{n} x_{i} W_{i} g\left(\left\|y_{0}-x_{i}\right\|^{2}\right)}{\sum_{i=1}^{n} w_{i} g\left(\left\|y_{0}-x_{i}\right\|^{2}\right)}$ calculate the formula of new candidate $y$.

(3) Calculate the similarity of the candidate target with $y$ target model:

$\rho[\mathrm{p}, q(y)]=\sum_{u=1}^{m} p_{u} q_{u}(y)$

(4) When $\left\|y-y_{0}\right\| \prec \varepsilon$, the iteration is stopped, otherwise go to step (2). 


\section{Hybrid tracking algorithm}

The paper calculates the similarity $\rho\left[\mathrm{p}, q\left(y_{0}\right)\right]$ of $\mathrm{y}_{0}$ and the target model, when $\rho\left[\mathrm{p}, q\left(y_{0}\right) \succ \theta\right]$, then the target motion model doesn't change. When $\rho\left[\mathrm{p}, q\left(y_{0}\right) \prec \theta\right]$, change motion model and predict the initial position, until $\rho\left[\mathrm{p}, q\left(y_{0}\right) \succ \theta\right]$.In the experiment, we take $\theta=0.8$. In order to verify the effectiveness of the proposed algorithm, we have tested a variety of different types of motion, different resolution of the sports video sequences, and have achieved good results. Table 1 is the technical parameters in the process of this paper. Figure 1- Figure 5 are the effect of the algorithm in this paper.

Table 1 The technical parameters of the test process

\begin{tabular}{|c|c|c|c|c|c|c|}
\hline Test video & Resolution & $\begin{array}{c}\text { Number of } \\
\text { frames }\end{array}$ & $\begin{array}{c}\text { Color } \\
\text { number }\end{array}$ & $\begin{array}{c}\text { Number of } \\
\text { samples }\end{array}$ & $\begin{array}{c}\text { Average } \\
\text { iteration }\end{array}$ & $\begin{array}{c}\text { Computational } \\
\text { time/ms }\end{array}$ \\
\hline Table Tennis & $352 * 288$ & 85 & $4 * 4 * 4$ & 100 & 6 & 3541 \\
\hline $\begin{array}{c}\text { Horizontal } \\
\text { bar }\end{array}$ & $560 * 381$ & 87 & $8 * 8 * 4$ & 200 & 4 & 5075 \\
\hline Football & $352 * 288$ & 81 & $8 * 8 * 8$ & 400 & 5 & 7250 \\
\hline Weightlifting & $720 * 576$ & 78 & $8 * 8 * 8$ & 400 & 4 & 12920 \\
\hline
\end{tabular}

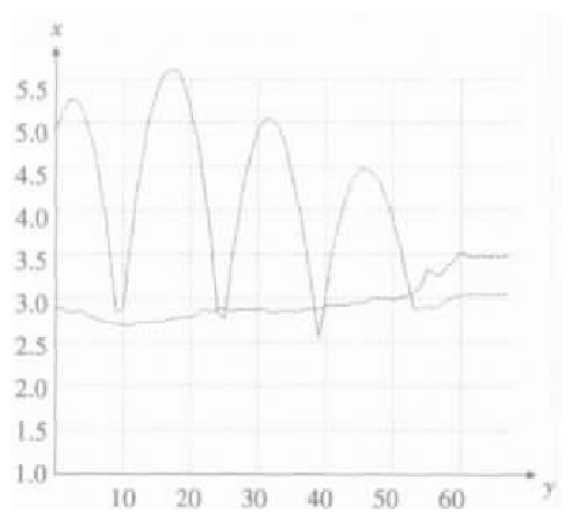

Figure 1 Table tennis tracks trajectory diagram

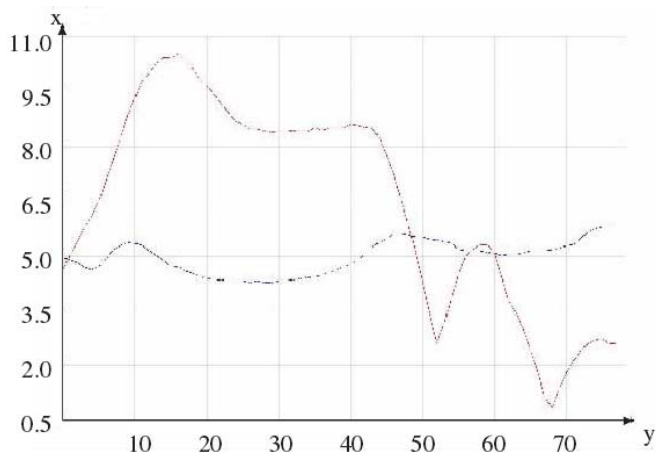

Figure 2 Barbell tracks trajectory diagram

\section{Conclusions}

In this paper, a new real-time target tracking algorithm is proposed for sports video, and a good result is achieved. Target tracking in sports video is very different from the traditional video tracking. The traditional video tracking is mainly used for target monitoring, the moving speed is slow, and the movement pattern will not change greatly. Therefore, the traditional target tracking algorithm is 
difficult to achieve good results, and the algorithm in the paper is mainly used to improve the prediction accuracy of the moving target by using the particle filter, which ensures that the mean shift algorithm converges to the local optimal solution.

\section{References}

[1] Katja NUmmiaro, Esther Koller- Meier, Luc Van Gool. A Color- based Particle Filter [J].Image and Vision Computing, 2002, (20):35-37.

[2] Ma Bo, Zhang Tianwen. A novel contour tracking algorithm[J]. Signal processing. 2004, 2 (20):123-125.

[3] Dorin Comaniciu, Peter Meer.Kernel- based Object Tracking [J].IEEE transactions On pattern analysis and machine intelligence, 2003, 25(5):309-311.

[4] Sanjiv Singh, Paul Keller. Obstacle Detection for High Speed Autonomous Navigation, Robotics and Automation [C]//1991 IEEE International Conference on 3:2798-2805.

[5] Criminisi, P. Perez. Region Filling and Object Removal by Exemplar based Image In painting [J]. IEEE Transactions on

[6] Marcelo Bertalmio, Luminita Vese. Image Filling-in in a Decomposition Space [J]. IEEE, 2003, (1):851 -853.

[7] Rogers D F.Procedure Elements for Computer Graphics [M].New York: McGraw-H ill, 1985.

[8] Bian ZQqi, Zhang Xuegong. Pattern recognition[M]. Beijing: Tsinghua University press, 2000: 280. 